\title{
Formação de imagens na óptica geométrica por meio do método gráfico de Pierre Lucie
}

\author{
Image formation in the geometrical optics by means of the Pierre Lucie's graphical method
}

\author{
Fábio F. Barroso ${ }^{1}$, Silvânia A. Carvalho*2, José A. O. Huguenin ${ }^{3}$, Alexandre C. Tort ${ }^{4}$ \\ ${ }^{1}$ Instituto de Ciências Exatas, Programa de Pós-Graduação em Ensino de Física, Universidade Federal Fluminense, Polo de \\ Volta Redonda, Volta Redonda, RJ, Brasil. \\ ${ }^{2}$ Departamento de Matemática, Física e Computação, Universidade do Estado do Rio de Janeiro, Campus de Resende, \\ Resende, RJ, Brasil. \\ ${ }^{3}$ Instituto de Ciências Exatas, Universidade Federal Fluminense, Campus de Volta Redonda, Volta Redonda, RJ Brasil. \\ ${ }^{4}$ Instituto de Física, Universidade Federal do Rio de Janeiro, Rio de Janeiro, RJ, Brasil.
}

Recebido em 11 de Abril, 2017. Revisado em 26 de Junho, 2017. Aceito em 25 de Julho, 2017.

\begin{abstract}
Neste trabalho resgatamos e modernizamos o método gráfico de Pierre Lucie (1917 - 1985) para o estudo de formação de imagens a partir de lentes delgadas, no contexto de óptica geométrica. Associamos a este método o uso de Tecnologia de Informação e Comunicação (TIC) por meio do simulador PhET - Simulações Interativas, para visualização da formação de imagens e obtenção de pontos conjugados, bem como o uso do software GeoGebra para analisar os dados e chegar à equação de Gauss. Tendo por base a Teoria de Aprendizagem Significativa de Ausubel, uma sequência didática para ensino de formação de imagens por lentes delgadas foi elaborada e aplicada em turmas do ensino médio. Os resultados da aplicação são também apresentados neste trabalho e indicam que o uso de TIC associada ao método gráfico de Pierre Lucie pode ser potencialmente significativo.
\end{abstract}

Palavras-chave: física e seu ensino; tecnologias de informação e comunicação.

In this work we recover and update a graphical method introduced by Prof. Pierre Lucie (1917 - 1985) in the study of image formation by thin lens. The method is associated by us with the modern technologies of information and communication through a PheT simulation that allows the visualization of image formation and the acquisition of the conjugated points. We also make use of the dynamical geometry software GeoGebra to analyze the data and obtain Gauss' equation for conjugated points. A class-oriented procedure for the teaching of image formation by thin lens was prepared and applied at the high-school level. Results indicate that this updated graphical method may be potentially significant.

Keywords: Physics and its teaching; information technologies.

\section{Introdução}

O estudo da óptica tem um papel importante atualmente, seja pela natureza da luz, de riqueza conceitual fantástica com aplicações em inúmeras tecnologias baseadas no laser (acrônimo para "amplificação da luz por emissão estimulada da radiação", em inglês), seja por permitir a compreensão de importantes instrumentos de observação, começando pelo olho humano, formado por um sistema óptico adaptativo de alta performance, chegando a microscópios ópticos utilizados em vários campos do conhecimento. Tais instrumentos são baseados na formação de imagens por lentes, tema comumente classificado como pertencente à óptica geométrica. A formação de imagens por lentes convergentes é um tópico recorrente em vários livros didáticos do ensino médio e tem sido tema de investigações que aprofundam a discussão, como por exemplo o estudo de imagens tridimensionais [1], no

*Endereço de correspondência: silvania.carvalho@uerj.br campo mais fundamental, e a investigação de descontinuidades de imagens feitas por lupas [2], no campo de instrumentação.

O ensino de óptica geométrica tem recebido constante atenção de forma a responder aos desafios do ensino de física: carga horária cada vez mais reduzida e uma nova geração de alunos que cresce em meio a novas tecnologias. A influência de experimentos demonstrativos no ensino de óptica geométrica no ensino médio foi investigada, mostrando bom aproveitamento dos alunos [3] para além dos ganhos proporcionados pela ludicidade intrínseca do uso de experimentos. O uso de experimentação, por outro lado, esbarra na carência de infraestrutura adequada na grande maioria das escolas brasileiras. Uma sequência didática foi concebida na literatura a partir da concepção de luz e visão trazidas pelos alunos, explorando concepções alternativas, a partir do conhecimento que os alunos trazem [4]. A valorização dos conhecimentos prévios é um dos pilares da teoria de aprendizagem 
significativa, de David P. Ausubel [5 6], que serve de base para várias propostas didáticas inclusive para ensino de óptica em cursos de graduação em física [7. Além disso, ao considerarmos a nova geração de alunos, o uso de tecnologias de informação e comunicação (TIC's) como elemento motivador para o aprendizado de física é fortemente recomendado 8.9] pois, de fato, o material didático com recursos tecnológicos torna atrativa a participação dos alunos, aumentando a pré-disposição ao aprendizado, além de aproveitar a facilidade dos estudantes com aplicativos e dispositivos tecnológicos; fazendo com que este material seja potencialmente significativo. Podemos citar a criação de hipertextos contendo simulações e animações voltadas ao ensino de óptica [10]. Para o estudo da formação de imagens com lentes convergentes basicamente se estuda a equação de Gauss [11] .

Neste trabalho, apresentamos o desenvolvimento de uma sequência didática para ensino de formação de imagens a partir do método de Pierre Lucie, um método alternativo, que utiliza o gráfico dos pontos conjugados desenvolvido pelo Prof. Lucie [12]. Na seção 2 apresentamos uma breve sumarização da Teoria da Aprendizagem Significativa e discutimos o uso de Tecnologia de Informação e Comunicação (TIC's) neste contexto. Uma breve biografia do Prof. Pierre Lucie é apresentada na Seção 3. A Seção 4 é dedicada a dedução da equação de Gauss a partir do método gráfico de Pierre Lucie e do método geométrico comumente encontrado na literatura. A Seção 5 é dedicada a apresentacção de uma sequência didática com o uso de Laboratório virtual (do aplicativo PhET, do inglês "Physics Educational Technology") e aplicativos de manipulação algébrica (GeoGebra). Por fim, concluímos o trabalho ressaltando os principais resultados obtidos assim como sugestões de aplicação.

\section{Aprendizagem Significativa e TIC's}

A Teoria da Aprendizagem Significativa, desenvolvida por David P. Ausubel [5], parte do princípio de que o processo ensino-aprendizagem ocorre a partir da transformação da estrutura cognitiva do aprendente, já que o conhecimento prévio trazido pelo aluno é encarado como um dos fatores que mais influencia no seu aprendizado. Estes conhecimentos prévios, ancorados na estrutura cognitiva do aprendente são chamados de subsunçores. A partir destes subsunçores [6], pela vivência do aluno, modelos mentais sobre o mundo, em particular sobre conceitos físicos, serão construídos na estrutura cognitiva do aprendente. No processo, segundo Ausubel, ocorre uma negociação de significados entre o novo conhecimento e os subsunçores existentes, que são modificados na estrutura cognitiva do aluno se o processo ensino-aprendizagem se deu de forma significativa, ou mesmo novos subsunçores são criados, portanto maior significado para o aprendente frente ao tema de estudo.

Outro ponto importante a ser considerado é que na Aprendizagem Significativa o aluno dá uma significado pessoal ao conhecimento, sendo adquirido de forma nãoliteral. Além disso, este novo conhecimento não interage com qualquer subsunçor, ou seja, a aprendizagem é dita não-arbitrária. Isso leva a dois outros fatores-chave no processo: o aluno, para dar significado ao aprendizado, deve apresentar uma pré-disposição ao aprendizado e, para que os subsunçores corretos sejam acessados, o material instrutivo ou a sequência didática utilizada deve apresentar organizadores prévios condizentes com o conceito a ser estudado, ou seja, deve ser potencialmente siginificativa 6 7]. Em suma, podemos elencar três fatores determinantes para que o aluno tenha uma aprendizagem significativa: (i) valorização do conhecimento prévio, (ii) pré-disposição do aprendente, e (iii) material potencialmente significativo.

Considerando a geração atual de estudantes, dita "geração digital", onde o uso de tecnologias de informação é amplamente utilizado, inclusive em áreas socialmente vulneráveis, o uso de TIC's pode ser uma ferramenta adequada por apresentar uma linguagem de fácil comunicação com os alunos, já que faz parte de um eixo de interesse do mesmo. Acreditamos que um material (em nosso caso uma sequência didática) que explore TIC's tem potencial para (i) contribuir para despertar interesse e pré-disposição ao aprendizado no aluno, e (ii) naturalmente ser plataforma de organizadores prévios no processo ensino-aprendizagem. Por este motivo, a escolha do uso de "laboratório virtual" como uma das plataformas da sequência didática apresentada.

\section{Breve biografia de Pierre Lucie (1917-1985)}

Pierre Henry Lucie nasceu em 14 de agosto de 1917 na Gasconha, França, e obteve seu Bacharelado em Filosofia e Matemática pela Universidade de Toulouse. Em 1937 ingressou na Escola Especial Militar de Saint-Cyr e, durante a segunda guerra mundial, foi mantido prisioneiro de 1940 à 1945. Durante o período em que ficou recluso dedicou-se a estudar Física. Imigrou para o Brasil onde inicialmente trabalhou como motorista de caminhão enquanto regularizava sua documentação. Quando sua documentação acadêmica foi finalmente oficializada, ministrou aulas de Física nos anos de 1950 no Colégio Santo Inácio e auxiliou na criação do instituto de Física da PUC-Rio. O professor Lucie foi um dos idealizadores e primeiro coordenador do ciclo básico do CTC (Centro Técnico Científico) operante já em 1961 com o nome de "Curso Fundamental" e formalizado pela instituição pouco antes da reforma universitária oficializada pelo MEC, em 1968 [12]. Participou, em 1963, da reformulação do ensino da Física nos Estados Unidos como membro do Physical Sciences Study Committee (PSSC) desenvolvido no Massachussets Institute of Technology (MIT). Este modelo de ensino foi implementado na PUC-Rio no mesmo ano e na Universidade de Brasília, em 1965. Sua convicção sobre a importância da dedicação de docentes 
e pesquisadores ao ensino fez dele membro atuante da comissão de especialistas em ensino de ciências do MEC, responsável pela organização curricular do ciclo básico universitário em todo o país, nos anos 1970. Em 1977, participou de forma decisiva na reformulação do ensino de Física no Instituto de Física Gleb Wataghin, da Universidade Estadual de Campinas. Pierre Lucie faleceu em 12 de setembro de 1985.

\section{O cálculo analítico: o método Pierre Lucie para lentes convergentes}

A Figura 1 mostra um esquema típico empregado para estudar a formação da imagem por meio de uma lente convergente. Aqui ele serve apenas para definir a notação: a reta $A B$ representa o tamanho do objeto, a reta $A^{\prime} B^{\prime}$ representa o tamanho da imagem, a distância do ponto $O$ até $F_{i}$ representa a distância focal da lente $f, p$ representa a distância do objeto à lente e $p^{\prime}$ a distância da imagem à lente.

O traçado de raios, apresentado neste trabalho, vale para raios paraxiais que atravessam uma lente convergente delgada. Consequentemente, podemos afirmar que $A B \approx C O \approx O$ e podemos desprezar o desvio lateral do raio que passa pelo centro da lente. Neste contexto, podemos utilizar a semelhança entre os triângulos $A B O$ e $A^{\prime} B^{\prime} O$ e $C O F_{i}$ e $A^{\prime} B^{\prime} F_{i}$ que fornece respectivamente

$$
\frac{|A B|}{\left|A^{\prime} B^{\prime}\right|}=\frac{|O B|}{\left|O^{\prime} B^{\prime}\right|} \Rightarrow \frac{O}{I}=-\frac{p}{p^{\prime}}
$$

e

$$
\frac{f}{f-p^{\prime}}=\frac{O}{I} \quad \Rightarrow \quad \frac{f}{f-p^{\prime}}=-\frac{p}{p^{\prime}},
$$

para obtermos a equação de Gauss $1 / f=1 / p+1 / p^{\prime}$. Veremos, com maior rigor matemático, a obtenção da equação de Gauss através do método geométrico na próxima seção.

Uma maneira de deduzirmos a equação de Gauss dos pontos conjugados é por meio de um método que envolve elementos simples geometria analítica. Considere a Figura

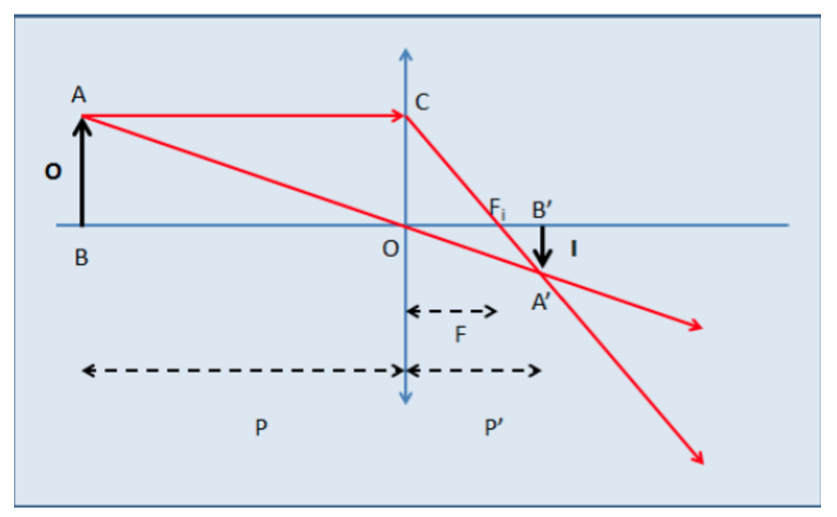

Figura 1: Esquema geralmente adotado para a obtenção da equação de Gauss.
2 (a) que ilustra a situação para a qual queremos encontrar a equação da reta que passa pelos pontos $P$ e $P^{\prime}$.

A equação geral de uma reta é dada por:

$$
y=a x+b,
$$

onde $a$ é o coeficiente angular e $b$ é o coeficiente linear. Juntamente com a equação acima, devemos levar em conta as coordenadas cartesianas dos pontos destacados do gráfico dadas por

$$
\begin{aligned}
& F=(0, f) ; \quad F^{\prime}=(f, 0) ; \quad O=(0,0) \\
& P=(0, p) \text { e } P^{\prime}=\left(p^{\prime}, 0\right)
\end{aligned}
$$

Neste caso, para determinar os coeficientes angular e linear da equação da reta, Eq. (3), substituímos as coordenadas $x=0$ e $y=p$ referentes ao ponto $P=(0, p)$
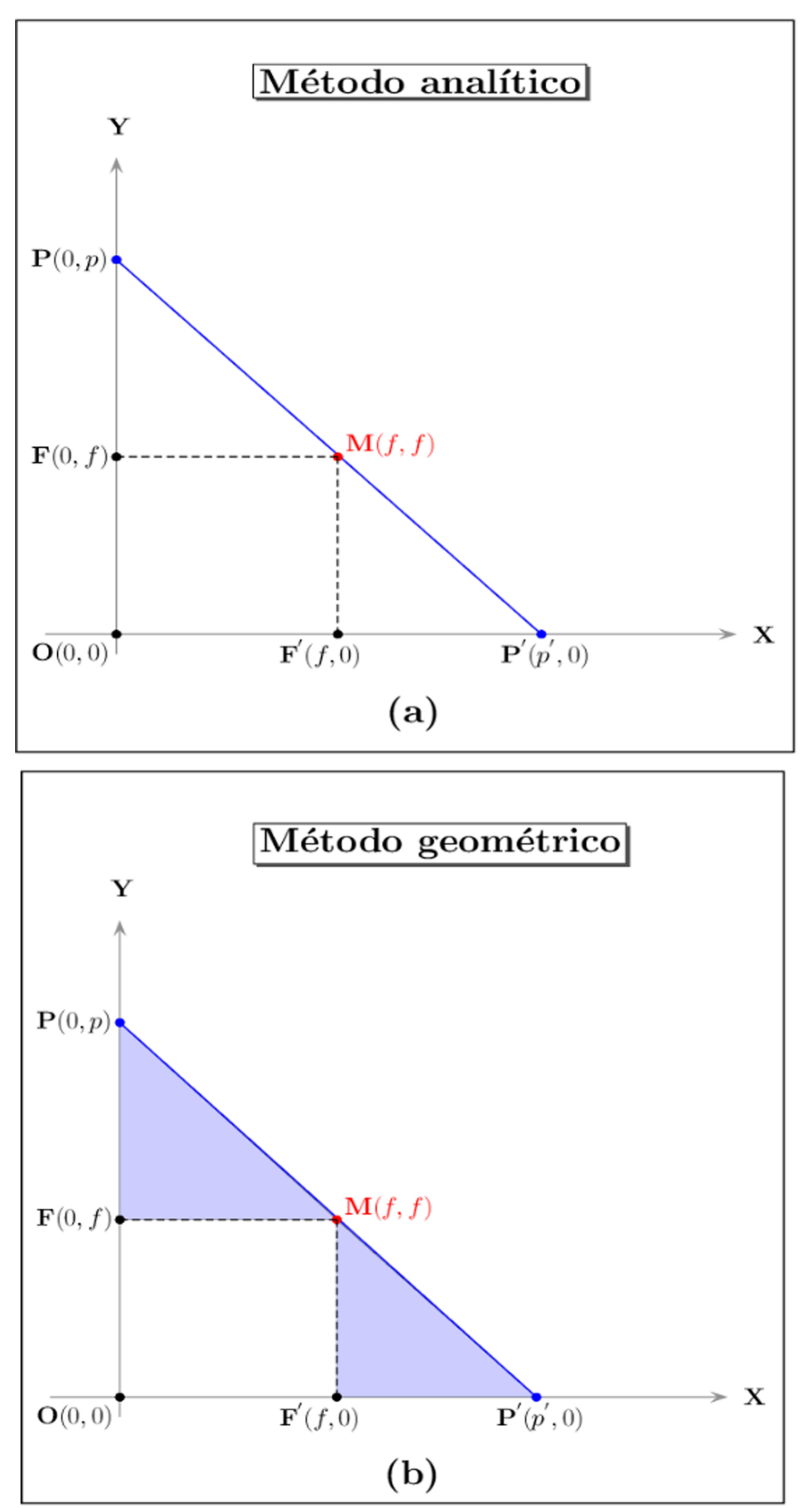

Figura 2: Gráficos ilustrando os pontos utilizados na obtenção da equação de Gauss. 
para obter

$$
p=a \cdot 0+b \Rightarrow b=p .
$$

Ao considerarmos o ponto $P^{\prime}=\left(p^{\prime}, 0\right)$, chegamos a relação

$$
0=a \cdot p^{\prime}+p \Rightarrow a=-\frac{p}{p^{\prime}} .
$$

Substituindo os valores de $a$ e $b$ acima na Eq. (3), chegamos a equação da reta

$$
y=-\frac{p}{p^{\prime}} x+p .
$$

A última etapa da dedução consiste em usar as coordenadas do ponto $M=(f, f)$ que leva a expressão

$$
f=-\frac{p}{p^{\prime}} f+p,
$$

que pode ser simplificada de modo a fornecer

$$
f=\frac{p}{1+\frac{p}{p^{\prime}}}=\frac{p p^{\prime}}{p+p^{\prime}} .
$$

A partir de manipulações algébricas simples podemos reescrever a equação acima como

$$
\frac{1}{f}=\frac{1}{p}+\frac{1}{p^{\prime}}
$$

que é a equação de pontos conjugados de Gauss. É importante ressaltar que o gráfico não representa a situação física real. Ele apenas representa um método matemático alternativo. Por esta razão, o ponto $M$ não deve ser chamado de distância focal. Este ponto está relacionado as coordenadas $(f, f)$ cujo valor é igual a $f$ tanto nos eixos das coordenadas quanto das abscissas.

Uma das vantagens deste método é que podemos utilizar as coordenadas dos pontos associados a posição do objeto e da imagem, marcadas no papel milimetrado ou lançadas no GeoGebra, para extrair informação sobre o foco da lente, ou seja, conectamos dados experimentais a uma informação prévia da lente que geralmente é fornecida pelo fabricante. Além disso, utilizamos uma simulação do $P h E T$ para obter dados que podiam ter sido obtidos por meio de um experimento mais complexo e que poderia requerer recursos difíceis de serem encontrados em um grande número de escolas.

\section{Método geométrico: semelhança de triângulos}

O método apresentado na seção anterior pode ser utilizado para substituir o método comumente empregado pelos professores de ensino médio em que a similaridade de triângulos leva a obtenção da equação de Gauss. Este cálculo baseia-se na dedução que considera, por exemplo, a semelhança entre os triângulos $\triangle F P M$ e $\Delta F^{\prime} M P^{\prime}$ da figura 2 (b) e que leva à relação

$$
\triangle F P M \approx \Delta F^{\prime} M P^{\prime}
$$

que pode ser utilizada para obter a expressão

$$
\frac{|O P|-|O F|}{|O F|}=\frac{\left|M F^{\prime}\right|}{\left|O P^{\prime}\right|-\left|O F^{\prime}\right|} .
$$

Como os segmentos de reta $\left|M F^{\prime}\right|,|O F|$ e $\left|O F^{\prime}\right|$ são congruentes, isto é:

$$
\left|M F^{\prime}\right|=|O F|=\left|O F^{\prime}\right|,
$$

podemos reescrever a equação (12) na forma

$$
\frac{|O P|-|O F|}{|O F|}=\frac{|O F|}{\left|O P^{\prime}\right|-|O F|},
$$

que pode também ser escrita como

$$
\left(\left|O P^{\prime}\right|-|O F|\right) \times(|O P|-|O F|)=|O F|^{2} .
$$

Efetuando as multiplicações indicadas acima, simplificando e dividindo o resultado pelo fator $|O P|\left|O P^{\prime}\right||O F|$ chegamos a equação

$$
\frac{1}{|O F|}=\frac{1}{|O P|}+\frac{1}{\left|O P^{\prime}\right|}
$$

que é a equação dos pontos conjugados em linguagem geométrica 11].

\section{Sequência didática utilizando o método de Pierre Lucie em laboratório virtual}

Considerando as TIC's como uma linguagem potencialmente significativa, associada à carência de infraestrutura adequada na grande maioria das escolas brasileiras para a realização de experimentos, optamos por utilizar o banco óptico do $P h E T$ para obter as coordenadas dos pontos $P=(0, p)$ e $P^{\prime}=\left(p^{\prime}, 0\right)$ que representam a distância do objeto a lente e da imagem a lente, respectivamente. Este aplicativo é de fácil manuseio e possui uma interface como ilustrado na Figura 3. Vale destacar que em havendo possibilidade de acesso a um banco óptico, mesmo com material de baixo custo, as coordenadas podem ser obtidas experimentalmente. Neste trabalho, nossa intenção é apresentar uma alternativa à falta de infraestrutura, explorando aplicativos que facilitam a linguagem da atual geração de estudantes.

Por meio do posicionamento do mouse sobre o objeto podemos facilmente alterar a distância entre o objeto e a lente e verificar automaticamente o que acontece com a imagem. Na próxima seção, veremos em detalhes como o aplicativo é utilizado na atividade. Uma explicação detalhada do uso do aplicativo foi apresentada no roteiro do aluno 13 . 


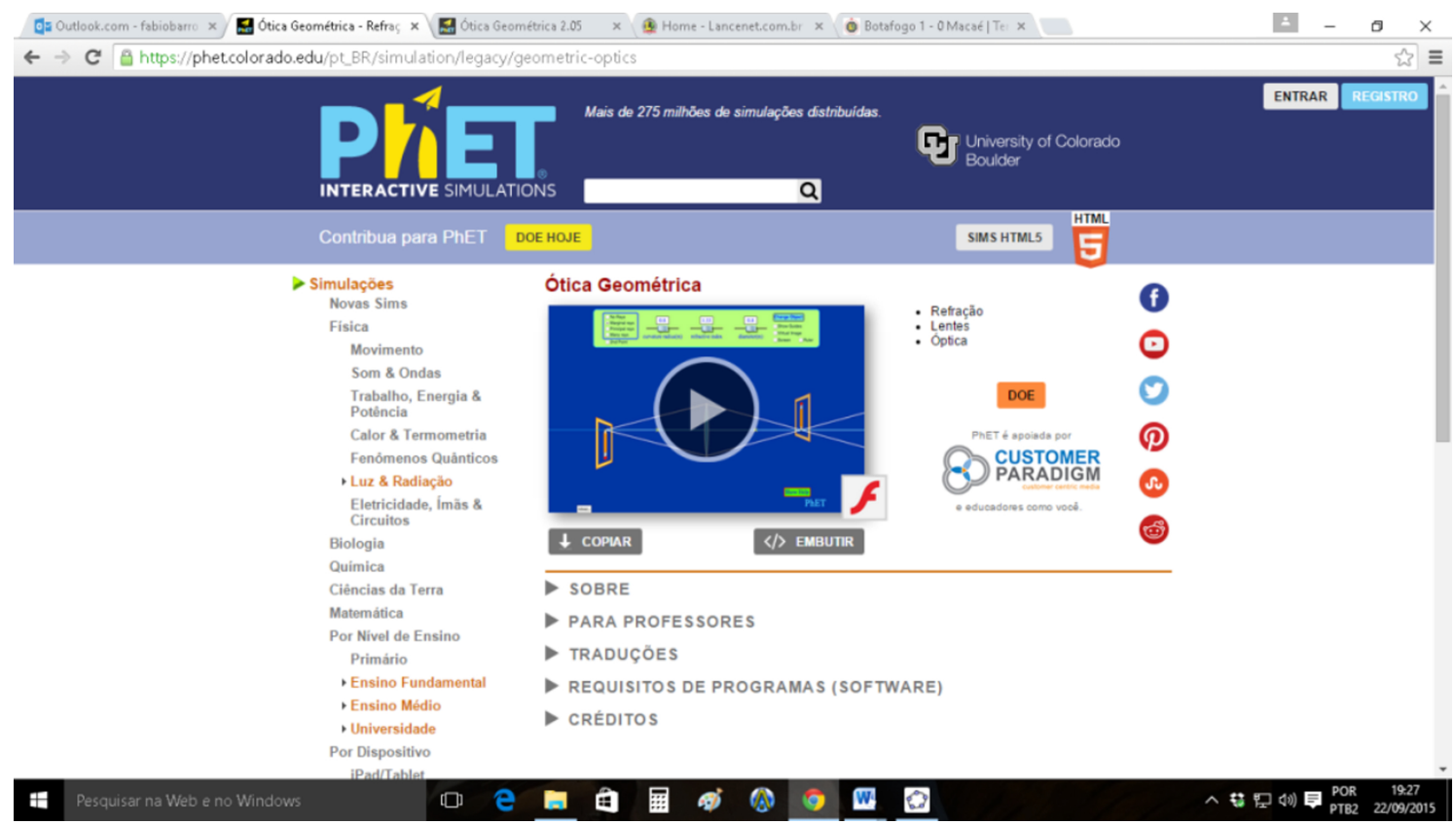

Figura 3: Figura ilustrativa do aplicativo PhET utilizado na primeira parte da atividade em aula (figura retirada do site https: //phet.colorado.edu/pt_BR/simulation/legacy/geometric-optics).

\subsection{A sequência didática por meio do método de Pierre Lucie}

A sequência didática proposta, na forma de produto educacional, bem como uma detalhada instrução de uso, pode ser encontrada na referência [13]. Trata-se de um roteiro de atividades a serem executadas com o auxílio do simulador de banco óptico. Para a aplicação deste material é importante a utilização de computadores com o simulador de banco óptico do PhET e o programa GeoGebra instalados. Como uma alternativa ao uso do GeoGebra pode ser fornecido papel milimetrado para os alunos. Caso a escola disponibilize de mais de um computador é interessante e recomendável que cada aluno utilize um computador de modo a valorizar a experiência pessoal do estudante.

Com relação aos conhecimentos prévios dos alunos [8], é recomendado que os alunos conheçam de maneira qualitativa os esquemas de formação de imagens nas lentes delgadas convergentes. Nas aulas anteriores à aplicação da sequência didática, o tópico de formação de imagens por meio de lentes esféricas delgadas deve ser apresentado, sendo construídos todos os casos de formação de imagens nas lentes delgadas convergentes e divergentes [11. É nesta etapa que se apresenta a refração e o desvio da luz. Uma abordagem interessante é proposta na referência [14], onde o conceito de refração é discutido no contexto de lentes esféricas. Com estes pré-requisitos, estamos aptos a aplicação da sequência didática com o método de Pierre Lucie.

No início da aula de aplicação do produto deve-se mostrar como o simulador funciona aos alunos, entrando na página https: //phet.colorado.edu/pt \penalty \@M \hskip \z@ skip \discretionary $\{-\}\{\}\{\} \backslash$ penalty $\backslash @ \backslash$ Mskip $\backslash z @$ skipBR/simulation/legacy/geometric-optics e mostrando as relações qualitativas de formação da imagem devido à mudança de posição do objeto em relação à lente que levam à mudança na distância da imagem até a lente (veja a Figura 4 .

Após esse período de adaptação com o simulador, que deve durar alguns minutos, deve-se pedir ao aluno que responda à primeira pergunta do roteiro: Você saberia responder o que é o foco de uma lente? Após os alunos refletirem por alguns minutos sobre a pergunta inicial, é solicitado que preencham a tabela 1 apresentada no roteiro do aluno colocando o objeto a 30, 45, 60 e 90 centímetros da lente esférica e que anotem as distâncias da imagem à lente em cada caso. Na etapa seguinte, a tabela preenchida será utilizada para aplicar o método gráfico das coordenadas, por meio da construção das três retas passando pelo pontos $P$ e $P^{\prime}$ no GeoGebra, como ilustrado na Figura 5.

Após a construção do gráfico, deve-se solicitar aos alunos para responderem as perguntas de 2 à 6 propostas no roteiro. Os últimos minutos de aula são reservados para demonstrar a equação dos pontos conjugados de Gauss a partir do gráfico formado no GeoGebra, como discutido na Seção 4.

Outro ponto importante na formação de imagens é questão da ampliação transversa destas. O uso do banco óptico virtual pode ser facilmente utilizado, uma vez que a régua do software pode fornecer os tamanhos do objeto e da respectiva imagem, a partir das quais pode-se discutir e definir a ampliação da imagem. 


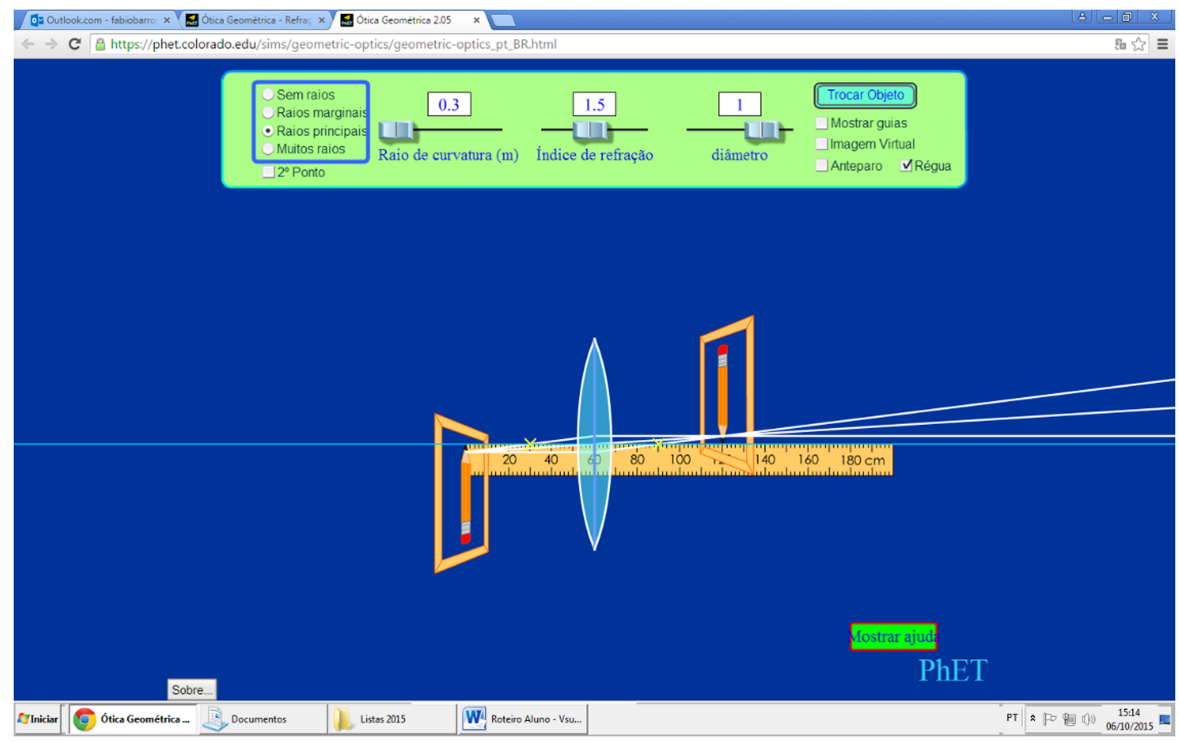

Figura 4: Tela do computador ilustrando a formação da imagem devido à mudança de posição do objeto em relação à lente.

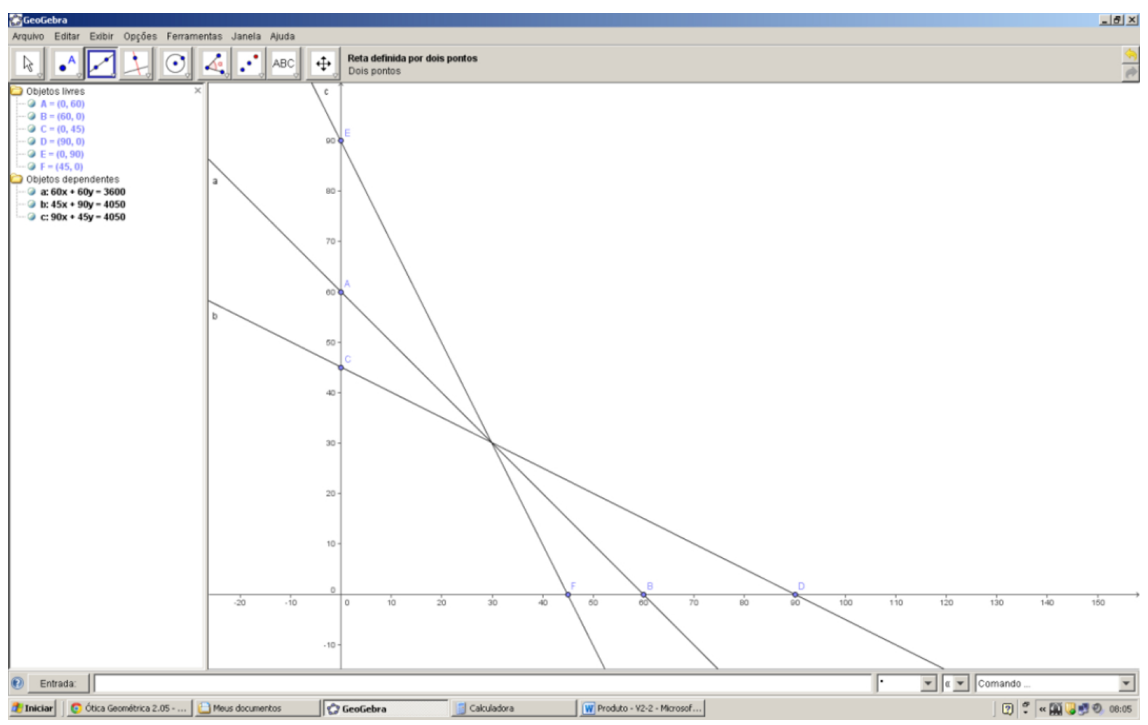

Figura 5: Tela do computador ilustrando o aplicativo GeoGebra utilizado para a obtenção das retas que passam por $P=(0, p)$ e $P^{\prime}=\left(p^{\prime}, 0\right)$.

Esse roteiro foi pensando para ser aplicado em uma aula de 50 minutos. Caso o professor disponha somente de um computador, deve escolher um aluno e orientá-lo na manipulação dos programas para que os demais em sala visualizem e tirem suas próprias conclusões. No caso em que o papel milimetrado for utilizado, o professor deve substituir o tempo de utilização do GeoGebra pela construção das retas no papel. Os roteiros do professor e do aluno foram preparados para auxiliar na aplicação do produto pelo professor e, ao mesmo tempo, levar o aluno a avançar na tarefa de modo independente.

\subsection{Aplicação e análise de resultados}

A metodologia de trabalho foi apresentada no início da aula, para que os alunos entendessem quais seriam as eta- pas da atividade. No momento da aplicação do produto educacional, os alunos foram levados para o laboratório de informática da escola onde estavam disponíveis cerca de vinte computadores para os estudantes e mais um computador ligado a um datashow disponível para o professor fazer projeções de sua tela para os estudantes. Nos computadores tínhamos: o arquivo do roteiro do aluno, o software GeoGebra instalado e conexão com a internet disponibilizada para os alunos acessarem o link do simulador de banco óptico do PhET. Foi pedido aos alunos para se dividirem em duplas e acessarem o PhET. Eles foram auxiliados na calibração do simulador assim como na etapa de simulação através da projeção de imagens em uma tela que podia ser visualizada pelos alunos. Depois 
de realizada a calibração, foi solicitado para os alunos seguirem o roteiro.

Em seguida, o GeoGebra foi projetado na tela e o software foi apresentado. Neste ponto, os alunos foram instruídos a continuar o roteiro até o final, respondendo cinco perguntas. Por fim, as fichas impressas preenchidas foram recolhidas e a demonstração da equação de Gauss com o método gráfico das coordenadas foi apresentada. Os alunos que já tinham estudado espelhos esféricos ficaram impressionados com o método para se chegar à equação de Gauss, pois já conheciam a equação devido ao estudo dos espelhos esféricos. Durante a aplicação do produto alguns alunos relataram dificuldades em utilizar a régua do aplicativo $P h E T$ pois ela apresenta um escala que varia de $20 \mathrm{em} 20 \mathrm{~cm}$ e cada traço da régua mede $2 \mathrm{~cm}$, deixando o aluno com dúvidas no momento de realizar suas medidas. Neste caso, surge a oportunidade da discussão de erro experimental na medida realizada devido ao instrumento de medida utilizado. Outra observação importante sobre o PhET é que o aplicativo não constrói a imagem virtual do objeto e este ponto poder ser abordado com os alunos.

$\mathrm{Na}$ aplicação deste produto educacional em uma escola com menos recursos, a substituição do software GeoGebra pelo papel milimetrado é necessária. Desta forma, o próprio aluno realiza a construção do método gráfico das coordenadas. Para se aplicar o produto educacional com papel milimetrado deve-se dispor de um computador com o aplicativo PhET, um projetor (Data Show ou TV) e folhas de papel milimetrado. Nesta etapa de aplicação, os alunos ficaram em uma sala de aula que dispunha de um computador com acesso à internet e uma televisão de 50 polegadas para as projeções. Foi entregue aos alunos um roteiro do aluno e uma folha de papel milimetrado. O aplicativo PhET foi projetado na televisão e um aluno foi convidado para realizar as medidas de $P$ e $P^{\prime}$. Outros alunos também demonstraram interesse em realizar as medidas o que foi permitido ao longo da aula. Esta atividade permitiu uma maior interação e discussão entre os alunos. A aplicação foi realizada em uma escola privada porque não houve tempo suficiente para aplicar em escolas da rede estadual por causa das greves e paralizações, no entanto, aplicamos o produto com o uso do papel milimetrado em algumas turmas para simular como seria a aplicação em uma escola com menos recursos.

Após a análise dos questionários, contidos no roteiro e respondidos pelos alunos, foi realizada uma comparação entre as duas estratégias de aplicação. Um total de 107 questionários provenientes de 8 turmas de ensino médio foram respondidos por 161 alunos alunos através da aplicação com GeoGebra, e 23 questionários provenientes de 2 turmas foram respondidos por 50 alunos através da aplicação com papel milimetrado.

Com relação a análise dos dados, observamos que a resposta referente à primeira pergunta Você saberia responder o que é o foco de uma lente? visou verificar o conhecimento prévio do aluno sobre o que é o foco de uma lente esférica. Nesta análise, foi encontrado um grande número de respostas satisfatória e parcialmente satisfatória, como ilustra a Figura 6

Já a segunda e a terceira perguntas: $O$ que aconteceu com as características da imagem quando você diminuiu a distância entre o objeto e a lente delgada de $60 \mathrm{~cm}$ para $45 \mathrm{~cm}$ ? e $O$ que aconteceu com as características da imagem quando você aumentou a distância entre o objeto e a lente delgada de $60 \mathrm{~cm}$ para $90 \mathrm{~cm}$ ? trouxeram informações sobre a leitura realizada através do aplicativo PhET, com uma maior percentagem de acerto no caso em que o papel milimetrado foi utilizado. Este aumento na percentagem de acerto se deve ao fato dos alunos interagirem mais na aplicação com papel milimetrado.

No caso em que a quarta pergunta Ao colocar o objeto a $30 \mathrm{~cm}$ da lente esférica, quais as características da imagem formada? foi abordada, houve uma menor percentagem de acerto, com resultados melhores para a aplicação com papel milimetrado. Esta mudança pode ter ocorrido porque os alunos discutiram os resultados obser-

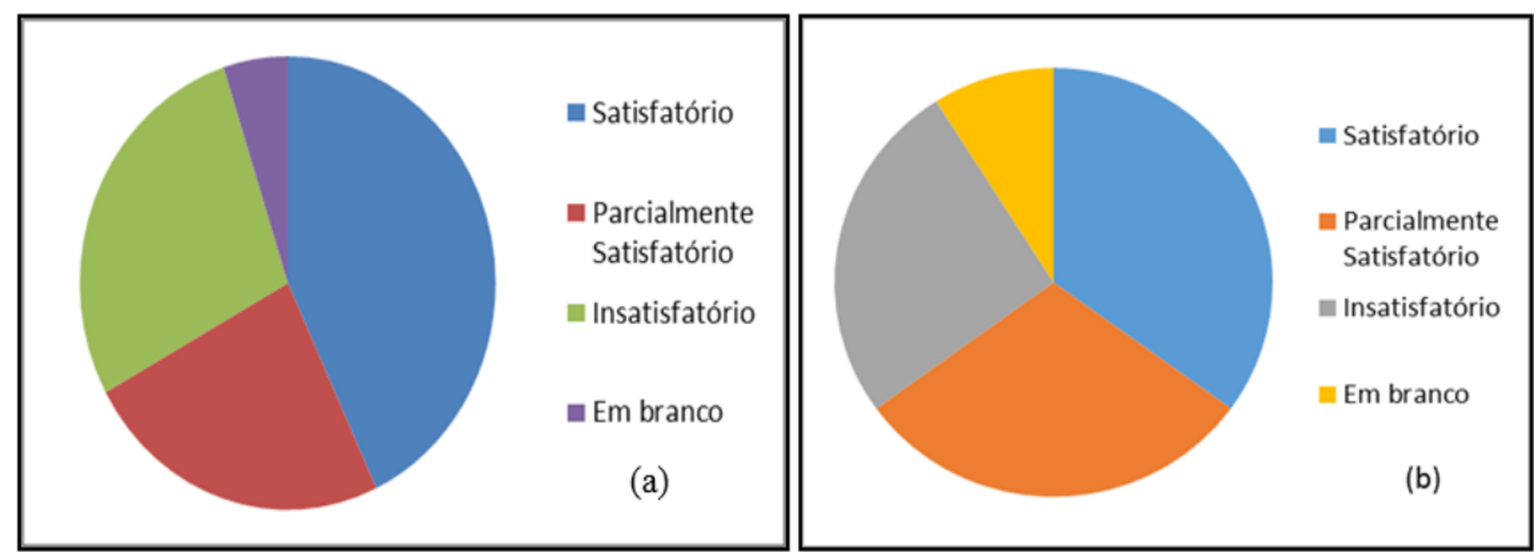

Figura 6: Gráficos em formato de pizza ilustrando os resultados referentes à pergunta 1 para aplicaçção com GeoGebra em (a) e papel milimetrado em (b). Nos itens (a) e (b), as percentagens de respostas satisfatórias, parcialmente satisfatórias, insatisfatótrias e em branco são, respectivamente, $43 \%, 24 \%, 28 \%$ e $5 \%$ para aplicação com o GeoGebra e $35 \%, 30 \%, 26 \%$ e $9 \%$ para o caso com papel milimetrado. 
vados na tela da televisão, mas não conseguiram conectar o resultado observado com as informações fornecidas na aula anterior à aplicação do produto.

$\mathrm{Na}$ etapa seguinte, a quinta pergunta Ao modificar a distância entre o objeto e a lente você acredita ter alterado alguma característica da lente esférica? veio reforçar conhecimentos prévios dos alunos, o que foi confirmado devido à alta percentagem de acertos em ambas as aplicações. Por fim, a sexta pergunta Observe o ponto de intercessão entre as retas. O que esse ponto significa? fez os alunos refletiram sobre uma característica da lente que foi discutida no início da aula. O resultado mostrou que grande parte dos alunos entendeu o que era este ponto e respondeu que era o foco da lente. Sendo possível observar a mesma percentagem de acerto em ambas as abordagens, como ilustrado na Figura 7, e um aumento na percentagem de acerto se comparamos as Figuras 6 7 .

O resultado da aplicação através dos dois métodos levou a maioria dos alunos a concluir qual era o significado do ponto de intercessão entre as retas e que este não muda com a posição do objeto. Além disso, os alunos conectaram um conhecimento prévio com os dados obtidos pela simulação e, a partir dos resultados, foi possível perceber que a aplicação com papel milimetrado proporciona maior interação entre os alunos e o método com GeoGebra proporciona uma reflexão mais individualizada que explora a habilidade dos mesmos com as tecnologias de informação e comunicação (um ou dois alunos por computador).

Outro fator importante que demonstra o resultado positivo do trabalho foi o relato de um professor que aplicou o produto em algumas turmas e disse: "Achei eficiente o uso da simulação do PhET como um recurso didático de construção do conhecimento de conceitos de físicos, apesar de nunca ter trabalhado com o software GeoGebra, esse recurso se mostrou muito interessante e de fácil manuseio. O manual de aplicação foi suficientemente claro para aplicação do produto, seguindo as instruções, consegui aplicar e a aula ficou muito interessante no ponto de vista didático.”.

\section{Conclusões}

Neste trabalho, resgatamos o método gráfico de Pierre Lucie através de duas demonstrações que exploram, respectivamente, aspectos anaíticos e geométricos para obtenção da equação de Gauss. Apresentamos uam breve biografia do Prof. Pierre Lucie, que fez contribuições importantes ao ensino de Física no Brasil com destaque internacional.

Também apresentamos uma sequência didática para o ensino médio fundamentada na Teoria de Aprendizagem Significativa de Ausubel, que explora o método de Pierre Lucie através do uso de Tecnologias de Comunicação e Informação (TIC's). O produto proveniente deste trabalho utilizou a manipulação de um banco óptico virtual do portal PhET associado ao uso de software GeoGebra para manipulação de dados. A sequência prevê, ainda, o uso de papel milimetrado em substituição ao GeoGebra, como uma sugestão para o caso de escolas que tenham menos recursos computacionais. Ao longo do trabalho, através da análise destes dados, foi possível chegar à equação de Gauss para lentes esféricas.

Durante a aplição da sequência foi possível observar uma participação efetiva maior, comparada à participação dos alunos em aulas tradicionais, discursivas, o que revelou o potencial da sequência para melhorar a pré-disposição dos alunos ao aprendizado, requisito essencial para a Aprendizagem Significativa. A análise dos dados apresentados nas Figuras 6 e 7 demonstrou que grande parte dos alunos entendeu que o ponto de interseção entre as retas era o foco da lente. Como pode ser visto, a noção prévia da distância focal possibilitou
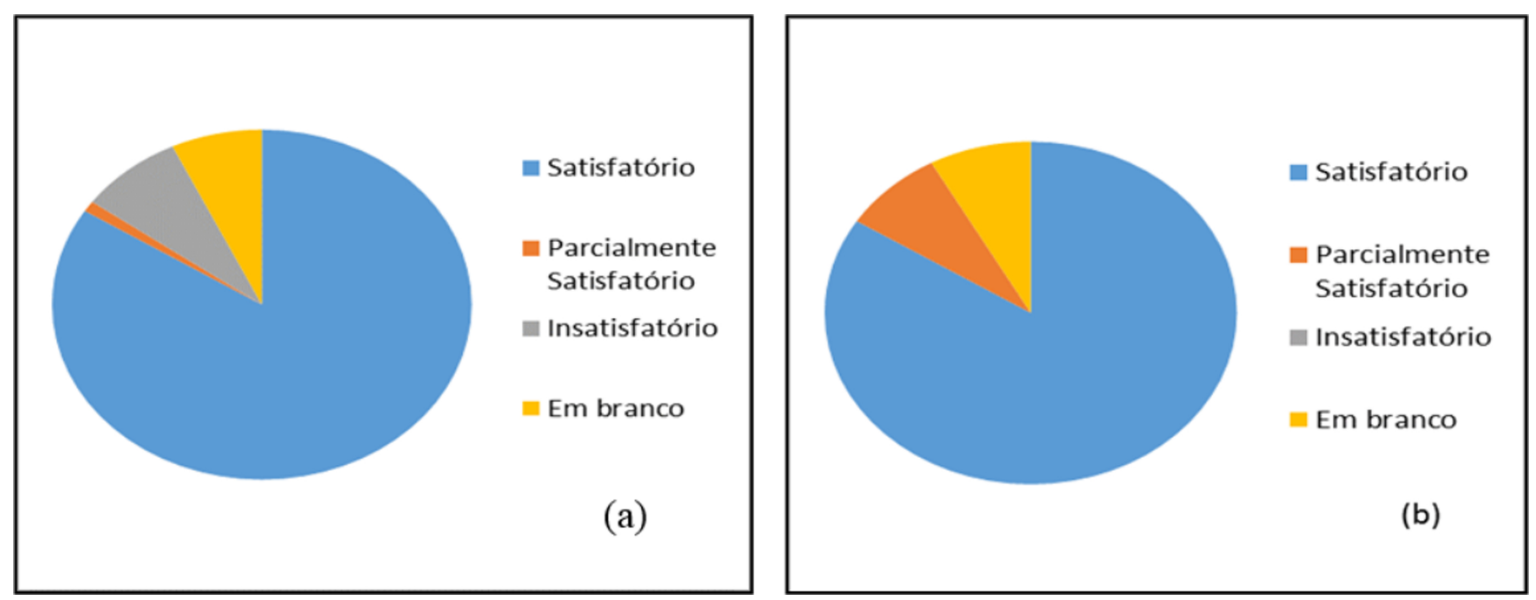

Figura 7: Gráficos em formato de pizza ilustrando os resultados referentes à pergunta 6 para aplicaçção com GeoGebra em (a) e papel milimetrado em (b). No item (a) e (b), as percentagens de respostas satisfatórias, parcialmente satisfatórias, insatisfatótrias e em branco são, respectivamente, $84 \%, 1 \%, 8 \%$ e $7 \%$ para aplicação com o GeoGebra e $84 \%, 8 \%, 0 \%$ e $8 \%$ para o caso com papel milimetrado. 
termos cerca de $60 \%$ de respostas satisfatórias e parcialmente satisfatórias (Figura 6), ao passo que após a realização de atividades da sequência este entendimento supera os $90 \%$ (Figura 7). Isto nos leva a crer que a sequência é potencialmente significativa.

Esta sequência didática foi desenvolvida durante a realização de uma dissertação de mestrado profissional no Polo 15 do MNPEF (ICEx - UFF). O produto educacional resultante, com instruções de aplicação detalhadas, pode ser encontrado encontrado na referência [13].

\section{Referências}

[1] Eden V. Costa e Lucia C. Almeida, Revista Brasileira de Ensino de Física 27, 231 (2005).

[2] Fernando L. da Silveira e Rolando Axt, Revista Brasileira de Ensino de Física 28, 421 (2006).

[3] Jair L.P. Ribeiro e Maria de Fátima da Silva Verdeaux, Investigações em Ensino de Ciências 18, 239 (2013).

[4] José P. Gircoreano e Jesúna Lopes de Almeida Pacca, Cad. Cat. Ens. Fís. 18, 26 (2001).

[5] M.A. Moreira e E.A.F. Masini, Aprendizagem Significativa: A Teoria de David Ausubel (Editora Moraes, São Paulo, 1997).

[6] M.A. Moreira, Teorias de Aprendizagem (EPU, São Paulo, 1999).

[7] Voltaire de O. Almeida e M.A. Moreira, Revista Brasileira de Ensino de Fírsica 30, 4403 (2008).

[8] Marcelo A. Pires e Eliane A. Veit, Revista Brasileira de Ensino de Física 28, 241 (2006).

[9] W.K. Adams et. al., Journal of Interactive Learning Research 19, 397 (2008).

[10] Valmir Heckler, Maria de Fátima Oliveira Saraiva e Kepler de Souza Oliveira Filho, Revista Brasileira de Ensino de Física 29, 267 (2007).

[11] Caio S. Calçada e Josão L. Sampaio, Fúsica Clássica $2^{a}$ ed (Atual, São Paulo, 1988).

[12] Suzana S. Barros, Pierre Lucie - Professor e Educador de Cientistas, Biografia (Editora UERJ, Rio de Janeiro, 2011)

[13] Fábio F. Barroso, Aplicação do Método Gráfico das Coordenadas de Pierre Lucie em uma Aula de Ensino Médio. Dissertação de Mestrado, MNPEF-ICEX/UFF (2016), disponível em http://www.sistcc.uff.br/sistcc/ _tcc/tcc_1481215521.pdf, acessado em 01/08/2017.

[14] Diogo Soga, Raul Dias Paiva Jr. e Mikiya Muramatsu, Revista Brasileira de Ensino de Física 39, 3506 (2017). 